Original

\title{
Carcinoma neuroendocrino de células grandes de pulmón. Análisis de una serie de once casos en un hospital universitario
}

\author{
R. García Gómez ${ }^{1}$ E. Álvarez Fernández², F. González Aragoneses 3 , M. Cebollero Presmanes ${ }^{2}$, N. Moreno ${ }^{3}$, \\ J. A. Arranz Arija, G. Abad, I. Siso, L. Iglesias, V. Pachón, V. Díaz, P. Khosravi, G. Pérez Manga ${ }^{1}$
}

\section{Resumen}

Propósito: Los tumores pulmonares con diferenciación neuroendocrina (DN) son un grupo heterogéneo de neoplasias que incluyen tumores carcinoides típicos, carcinoides atípicos, carcinomas neuroendocrinos de células grandes (CNCG) y carcinoma pulmonar de células pequeñas. Los CNCG constituyen menos del 5\% de los carcinomas pulmonares no células pequeñas (CPNCP). En este trabajo se describe una serie de CNCG, tratados en un solo centro a lo largo de 10 años.

Material y métodos: Se analizan 11 pacientes diagnosticados de CNCG (5 con histologías mixtas).

Resultados: La edad media de los pacientes fue de 66 años, 5 fueron varones, 4 tuvieron enfermedad localizada, 5 localmente avanzada y 2 diseminada. Siete pacientes, con tumores localizados, fueron tratados con cirugía radical, 2 de ellos recibieron quimioterapia adyuvante; 1 quimioterapia y radioterapia y los otros 3 solo quimioterapia. La mediana de supervivencia de la serie es de 24 meses, y la supervivencia global a 2 y 5 años del $45 \%$ y $27 \%$ respectivamente.

Conclusión: Los datos de nuestra serie corroboran las recomendaciones de que el manejo de los CNCG debe hacerse de forma similar al del resto de los CPNCP. La cirugía radical es el tratamiento fundamental en los tumores localizados. No hay datos suficientes que indiquen una peor respuesta a los tratamientos de quimioterapia o radioterapia en este tipo de tumores.

Palabras clave:

Carcinoma pulmonar. Células grandes. Diferenciación neuroendocrina.

Oncología, 2006; 29 (8):321-328

\footnotetext{
${ }^{1}$ Servicio de Oncología Médica

${ }^{2}$ Departamento de Anatomía Patológica

${ }^{3}$ Servicio de Cirugía Torácica

Hospital General Universitario Gregorio Marañón

Madrid (España)
}

Recibido: 27.01 .06

Revisado: 16.02 .06

Aceptado: 03.05.06 


\section{Summary}

Purpose: Lung carcinomas with neuroendocrine differentiation are a heterogeneous group of tumors related to typical and atypical carcinoids, neuroendocrine large-cell carcinomas (NLCC) and small-cell lung cancer (SCLC). NLCC comprises less than 5\% of non small-cell lung cancer (NSCLC). In this report, we describe a series of NLCC treated in a single institution in the last ten years.

Material and methods: Eleven patients diagnosed as having NLCC (5 of them with mixed histology).

Results: At diagnosis, the mean age of the patients was 66 years ( 5 males and 6 females); 4 had localized disease, 5 locally advanced disease, and 2 metastatic disease. Seven patients underwent initial radical surgery ( 2 of them followed by adjuvant chemotherapy), 1 patient received chemotherapy and radiotherapy, and 3 patients only chemotherapy. Median overall survival for the whole series was 24 months, and the overall 2-year and 5-year survival were $45 \%$ and $27 \%$ respectively.

Conclusion: Our data corroborate the general recommendation of treating NLCC in a similar way as the rest of the non small-cell lung cancer (NSCLC) are treated. Radical surgery is the main treatment for localized tumors. There are no data indicating a worse response of these tumors to radiation therapy or chemotherapy.

Key words: Large-cell lung cancer. Neuroendocrine differentiation.

\section{Introducción}

La clasificación histológica de los tumores epiteliales pulmonares, según la Organización Mundial de la Salud, incluye algunas tumoraciones de comportamiento benigno (Papilomas, adenomas, lesiones preinvasivas, etc) y otras, que representan la gran mayoría tanto por número como por incidencia, de desarrollo clínico generalmente maligno (Carcinomas de Células Escamosas, de Células Pequeñas, de Células Grandes, Adenocarcinomas, Carcinosarcomas, etc $)^{1}$. La compleja y heterogénea clasificación, que forman el segundo grupo, se suele simplificar y dividir en dos grandes categorías con evolución, pronóstico y tratamiento diferente ${ }^{2}$ :

A. Carcinoma de pulmón de no célula pequeña (CPNCP): escamosos, células grandes, adenocarcinomas y combinados.

B. Carcinomas de pulmón de células pequeñas (CPCP): "oat cell", células pequeñas/grandes, y combinado.

Sin embargo, esta simplificación no satisface plenamente la comprensión y posibilidades terapéuti- cas de un importante subgrupo de tumores malignos, que vienen a denominarse como tumores neuroendocrinos pulmonares (TNP). Los TNP son un grupo heterogéneo de neoplasias cuya clasificación se ha ido estableciendo lo largo de las últimas décadas, en base a criterios morfológicos por micoscopía óptica ${ }^{3,4}$, y otros datos de diferenciación neuroendocrina (DN) demostrables por inmunohistoquímica o microscopía electrónica ${ }^{5,6}$. Dentro de los TNP se incluyen tumores carcinoides típicos, tumores carcinoides atípicos, carcinomas neuroendocrinos de células grandes (CNCG) y carcinoma pulmonar de células pequeñas. Los Carcinoides Típicos (CT) representan TNP de bajo grado; los Carcinoides Atípicos (CA) TNP de grado intermedio; por último, los Carcinomas Neuroendocrinos de Células Grandes (CNCG) y los CPCP son TNP de alto grado. Esta heterogenicidad de los TNP implica diferencias clínicas, pronósticas, de tratamiento y de supervivencia de los pacientes ${ }^{7-11}$.

Los CNCG son poco frecuentes (menos del 5\% de los CPNCP), por lo que no existe suficiente información específica de esta entidad clínica. En el presente trabajo se describen distintos aspectos epidemiológi- 
cos, clínicos y de supervivencia en una serie de CNCG, diagnosticados y tratados en el H.G.U. "Gregorio Marañón" de Madrid en los últimos 10 años y se realiza una revisión de la literatura.

\section{Material y métodos}

Según datos del registro de tumores del Servicio de Anatomía Patológica en el H.G.U. "Gregorio Marañón" de Madrid, entre 1993 y 2003 se diagnosticaron 50 pacientes de CPNCP con DN. Once de ellos correspondían a CNCG. Se han registrado los datos de edad, sexo, tabaquismo, sintomatología, histología, localización tumoral y diseminación, estadificación clínica (analítica, TAC TA, Fibrobroncoscopia, etc) y estadificación postquirúrgica, acorde con el Sistema Internacional para la Estadificación del Cáncer de Pulmón ${ }^{12}$, así como del tratamiento y la supervivencia. El diagnóstico de CNCG en el espécimen obtenido se estableció en base a datos histológicos e inmunohistoquímicos, en general tras cirugía y/o por necropsia, y se realizó usando los criterios propuestos por Travis ${ }^{5}$, con tinción de hematoxilina-eosina de cortes de tejido embebido en parafina y fijados con formol. Dentro del panel de anticuerpos empleados, en general, para las neoplasias pulmonares se incluyen los siguientes marcadores neuroendocrinos: Cromogranina A (monoclonal NCL-CHROM, clon LK2H10, Dako, California, USA), Sinaptofisina (Policlonal, Biomeda, California, USA) y Leu-7 o CD57 (monoclonal, Clon HNK-1, Becton-Dickinson, California, USA). Estudios de microscopía electrónica no se detallan en este trabajo. La supervivencia fue medida desde la fecha de cirugía o desde el inicio del tratamiento médico hasta la muerte, o la fecha del último seguimiento. En dos pacientes fallecidos se practicó necropsia sistemática.

\section{Resultados}

Se analizan 11 pacientes diagnosticados de CNCG, cuyas características epidemiológicas y de estadificación se resumen en la Tabla I. Seis pacientes fueron diagnosticados de CNCG puros y los 5 restantes tuvieron histologías mixtas. El resto de datos histológicos se resumen en la Tabla II.
TABLA I

\section{Características de los pacientes}

\begin{tabular}{|lc|}
\hline $\begin{array}{l}\text { Número de pacientes } \\
\text { Edad (años) }\end{array} \quad 11$ \\
Media & \\
Extremos & 66 \\
Sexo & $52-77$ \\
$\quad$ Varones & 10 \\
Mujer & 1 \\
Tabaquismo & $100 \%$ (> 30 c/d) [30-60] \\
Clínica: & \\
Hallazgo & 3 \\
Síndrome constitucional & 3 \\
Dolor torácico & 2 \\
Neumonía & 2 \\
Cuadro Neurológico & 1 \\
Performance Status (Estado General): & \\
0 & 3 \\
1 & 5 \\
2 & 1 \\
3 & 2 \\
Estadio: & \\
I B & 3 \\
II B & 1 \\
III A* & 2 \\
IIIB & 1 \\
IV & 3 (Múltiples 2) \\
\hline
\end{tabular}

(*) 1 paciente tras quimioterapia neoadyuvante, en necropsia, se descubren metástasis hepáticas [E: IV].

Las Tablas III y IV resumen los tratamientos recibidos y la evolución de los pacientes según su estadio y tratamiento recibido. Es de destacar que 7 de los 11 pacientes fueron tratados exclusivamente con cirugía radical, y 2 de ellos, estatificados como IIIA recibieron quimioterapia adyuvante. El primero, sigue vivo y libre de enfermedad a los 78 meses, tras 3 ciclos de Carboplatino - Gemcitabina y posterior radioterapia sobre lecho quirúrgico y mediastino (55Gy). El otro paciente, se trató con 4 ciclos de Cisplatino y Vinorelbina y tuvo una supervivencia de 62 meses. Otros dos pacientes con enfermedad localmente avanzada, fueron tratados con quimioterapia de inducción. El primero de ellos, estadio IIIA y con serología positiva para HIV, recibió 2 ciclos de Cisplatino y Gemcitabina, obtuvo una respuesta parcial $^{13}$ y una supervivencia de 5 meses. Falleció por distress respiratorio secundario a neumonitis por citomegalovirus. En la necropsia se confirmó además la existencia de metástasis hepáticas no documentadas "in vivo". El segundo paciente, IIIB, se 
TABLA II

\section{Anatomía patológica}

\begin{tabular}{|c|c|}
\hline \multicolumn{2}{|l|}{ Histología: } \\
\hline Células Grandes (CG) & 6 \\
\hline Mixtos & 5 \\
\hline CG-Adenoc-Escam & 1 \\
\hline CG-Adenoc & 3 \\
\hline CG-Epider. & 1 \\
\hline \multicolumn{2}{|l|}{ Hallazgos microscópicos } \\
\hline Necrosis & $70 \%$ \\
\hline Fibrosis & $20 \%$ \\
\hline Abcesificación & $10 \%$ \\
\hline Calcificaciones & $10 \%$ \\
\hline Rosetas & $10 \%$ \\
\hline Elevado Ind. Mitótico & $30 \%$ \\
\hline \multicolumn{2}{|l|}{ Inmunohistoquímica } \\
\hline Cromogranina $+(30 \%)$ & $27,2 \%$ \\
\hline$+($ focal $)$ & $9 \%$ \\
\hline- & $63.6 \%$ \\
\hline $\mathrm{SF}+(40 \%)$ & $27,2 \%$ \\
\hline$+(10 \%)$ & $9 \%$ \\
\hline- & $63.6 \%$ \\
\hline Leu 7 - & $100 \%$ \\
\hline Necropsia & 2 \\
\hline
\end{tabular}

trató con 5 ciclos de Taxotere y Gemcitabina. Tras una respuesta parcial, se realizó radioterapia torácica (55Gy) y permanece libre de enfermedad a los 21 meses. Los dos últimos pacientes de la serie, que
TABLA III

\section{Tratamiento recibido}

\begin{tabular}{|lc|}
\hline Cirugía & $\mathbf{7}$ \\
Lobectomía Sup. I. & 2 \\
Lobectomía Inf. I. & 1 \\
Lobectomía Sup. D. / & 2 \\
Lobectomía - Parietectomía & 1 \\
Neumonectomía Izq. & 1 \\
Tratamiento adyuvante a Cirugía & $\mathbf{2}$ \\
Quimioterapia & 1 (Cis-P y VNR) \\
Quimioterapia y Radioterapia & 1 (Carbo y GZB) \\
Quimioterapia de inducción & $\mathbf{2}$ \\
Quimioterapia neoadyuvante & 1 \\
Quimioterapia y Radioterapia secuencial & \\
(neoadyuvante) & 1 \\
Sintomático & $\mathbf{2}$ \\
& \\
\hline
\end{tabular}

fueron tratados de forma paliativa-sintomática por mal performance status y enfermedad diseminada, fallecieron tras 1 y 2 meses respectivamente.

En el momento del análisis habían fallecido 8 pacientes. Cuatro de ellos, con estadios iniciales al diagnóstico (IB y IIB) fallecieron inesperadamente, dos pacientes por causas no tumorales (a los 4 y 24 meses), otro paciente por progresión tumoral a los 2 años y un último por segunda neoplasia después de 5 años libre de enfermedad. Otros 4 fallecidos, por

TABLA IV

Supervivencia

\begin{tabular}{|c|c|c|c|c|c|}
\hline \multicolumn{2}{|c|}{$\begin{array}{l}\text { Supervivencia mediana: } \\
\text { Rango: } \\
\text { Pacientes: }\end{array}$} & \multicolumn{4}{|l|}{$\begin{array}{l}2.5 \mathrm{a}(30.3 \mathrm{~m}) \\
1 \mathrm{~m}-99 \mathrm{~m}\end{array}$} \\
\hline$N^{o}$ & $E$ & $S$ & $C$ & Tto A/NA & Estado \\
\hline 1 & IIIA & 8a 3m (99m) & lsi & - & $(\mathbf{V})$ \\
\hline 2 & IIIA & $6 \mathrm{a} 6 \mathrm{~m}(78 \mathrm{~m})$ & lsi & [Qt A-RDT A] & $(\mathbf{V})$ \\
\hline 3 & IB & $2 \mathrm{a}(24 \mathrm{~m})$ & 1si & - & (F)M Adrenal \\
\hline 4 & IIIA & $14 \mathrm{~m}(14 \mathrm{~m})$ & lsd & - & (F)M Cer/Hep \\
\hline 5 & IB & $5 \mathrm{a} 2 \mathrm{~m}(62 \mathrm{~m})$ & $\mathrm{Ni}$ & [QtA] & (F)Neo Esofog \\
\hline 6 & IB & $2 \mathrm{a}(24 \mathrm{~m})$ & lsi & - & (F)Infarto Mio \\
\hline 7 & IIB & $4 \mathrm{~m}(4 \mathrm{~m})$ & lii & - & (F)Rotura Aort \\
\hline 8 & IV & $2 \mathrm{~m}(2 \mathrm{~m})$ & & {$[\operatorname{sintm}]$} & $(F)$ \\
\hline 9 & IV [Necro] & $1 \mathrm{~m}(1 \mathrm{~m})$ & & [sintm ] & (F) \\
\hline 10 & IIIA [IV- Necro] & $5 \mathrm{~m}(5 \mathrm{~m})$ & & [Qt NA (2c)] & (F)M Hepat \\
\hline 11 & IIIB & 1a $9 \mathrm{~m}(21 \mathrm{~m})$ & & [Qt $(5 c)+R D T]$ & (V) \\
\hline
\end{tabular}

(E) Estadio. (S) Supervivencia. (C) Cirugía. (Tto A/NA) Tratamiento Adyuvante/ Neoadyuvante a Cirugía. (V) Vivo. (F) Fallecido. a: años. m: meses. lsi: lobectomía superior izquierda. 1sd: lobectomía superior derecha. Ni: Neumonectomía izquierda. QtA: Quimioterapia adyuvante. RDT A: Radioterapia adyuvante. sintm: tratamiento sintomático. Necro: Necropsia. 


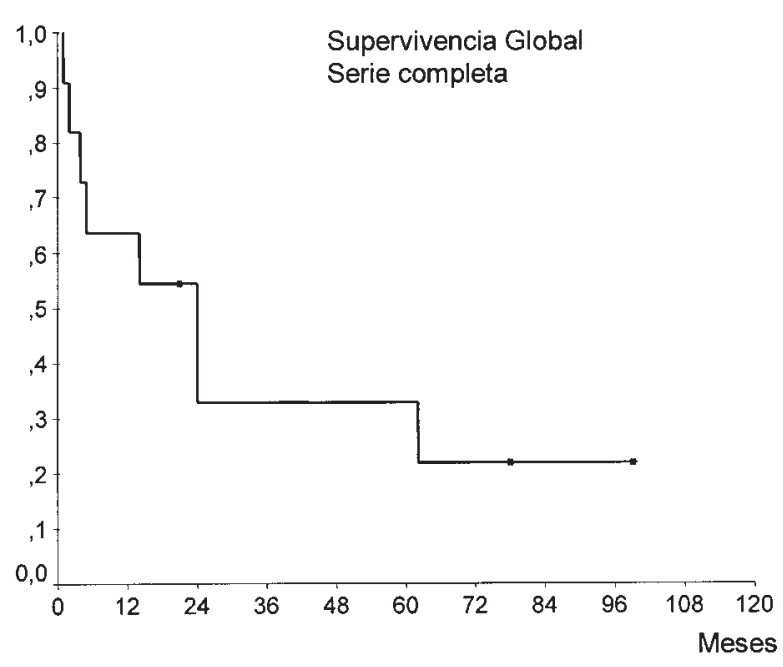

Figura 1. Supervivencia global de la serie completa de carcinomas neuroendocrinos de células grandes.

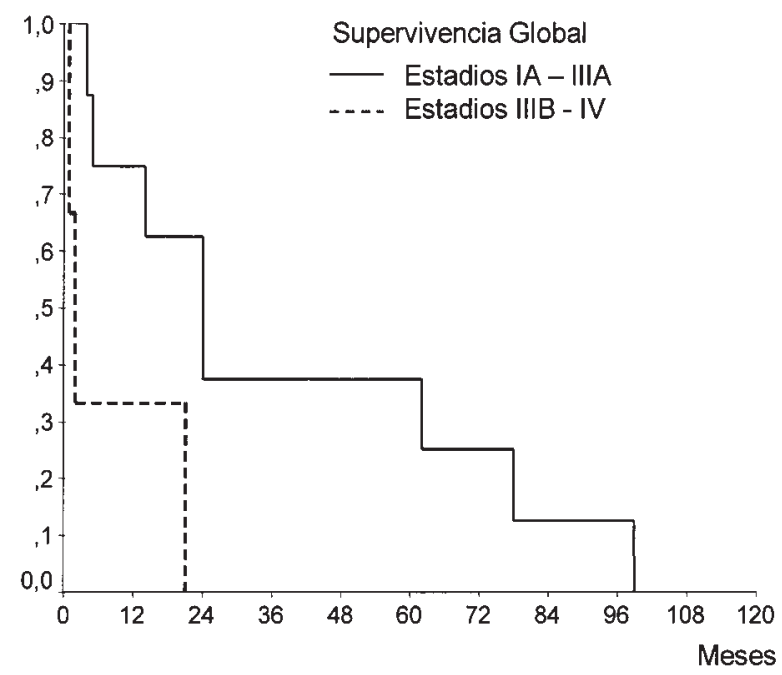

Figura 2. Supervivencia global de la serie completa según estadios al diagnóstico.

progresión de la enfermedad, incluyen dos pacientes que sólo recibieron tratamiento sintomático, el paciente con $\mathrm{HIV}+$, y otro en estadio IIIA. La supervivencia global de la serie es del $45 \%$ a 2 años y del $27 \%$ a 5 años y la mediana de supervivencia es de 24 meses (Figuras 1 y 2).

\section{Discusión}

El conocimiento de los TNP ha mejorado desde que en 1928 se describiera el primer caso $^{14}$. Desde entonces se han publicado diversas clasificaciones, todas ellas poco útiles. En las últimas décadas se ha ido estableciendo una clasificación más definitiva, en base a criterios morfológicos de microscopía óptica $^{3,4,15}$, inmunohistoquímica y microscopía electrónica ${ }^{5,6,15}$. Los TNP representan cerca de un 20\% de los carcinomas pulmonares y los CNCG tan sólo un $1-2 \%$ de los mismos ${ }^{10}$. Según algunos autores, los TNP forman un espectro continuo de una misma entidad, con alteraciones genómicas estructurales características, cuyas diferencias podrían ser más cuantitativas que cualitativas ${ }^{16}$. Por el contrario, otros opinan que los CNCG y CPCP derivarían de una stem-cell diferente a la de los CT. Dentro de estos tumores, los CNCG a su vez se subdividen en otras cuatro categorías: a) CNCG con morfología neuroendocrina y evidencia de diferenciación neuroendocrina por inmunohistoquímica y/o micoscopía electrónica; b) CNCG, sin datos morfológicos neuroendocrinos, aunque con evidencia de diferenciación neuroendocrina por inmunohistoquímica y/o micoscopía electrónica; c) CNCG con morfología neuroendocrina, sin evidencia de diferenciación neuroendocrina por inmunohistoquímica y/o micoscopía electrónica; d) CNCG sin morfología neuroendocrina y sin evidencia de diferenciación neuroendocrina por inmunohistoquímica y/o microscopía electrónica ${ }^{6}$. En este trabajo se analizan pacientes diagnosticados de CNCG que se ajustan a los supuestos a, b y c de los mencionados anteriormente.

Habitualmente no es fácil establecer la presencia de DN en los carcinomas de pulmón, sobre todo cuando la muestra biópsica es pequeña, o cuando se dispone únicamente de material citológico ${ }^{17}$. La diferenciación tumoral divergente es posible en la misma neoplasia, y así, la presencia de carcinoma epidermoide o de adenocarcinoma no necesariamente excluyen DN. Por otra parte ${ }^{17,18}$, para aceptar el diagnóstico de DN se recomienda el uso de un panel de anticuerpos monoclonales y además que haya reactividad positiva para dos o más de algunos de estos anticuerpos, como por ejemplo: cromogranina A, sinaptofisina, Leu-7 (CD-57), enolasa neuroespecífica, nuerotensina, creatinquinasa $\mathrm{BB}$, TTF1 etc ${ }^{17,19,20}$. Otros autores incluso recomiendan completar el diagnóstico con estudios de hibridación genómica. Pérdidas a nivel $5 q$ son frecuentes en TNP de alto grado y raras en otros TNP. Ganancias $5 q$ están presentes en el $25 \%$ de los CT y CA. 
Hay ganancias 3q en el 66\% de CPCP y es excepcional en otros CPNCP. Por último, la delección 11q está presente en el $55 \%$ de los $\mathrm{CA}^{21,22}$. Los pacientes analizados en este estudio, expresaron positividad para cromogranina y sinaptofisina en casi un $40 \%$ de los especimenes estudiados. El antígeno leu-7 fue en todos los casos negativo. Otros anticuerpos, que no se representan en la Tabla II, fueron también valorados, así como datos de microscopía electrónica. El diagnóstico se estableció tras cirugía en el $65 \%$ de los pacientes, por necropsia en el $10 \%$ $\mathrm{y}$, en el restante $25 \%$, la DN se detectó en la pieza de biopsia inicial. Estos datos son muy similares a los de otras series y, al mismo tiempo, ponen en evidencia la dificultad del diagnóstico preoperatorio de estos carcinomas ${ }^{23}$. En este trabajo no se efectuaron estudios cromosómicos ni de hibridación.

Desde un punto de vista epidemiológico, los CNCG son más frecuentes en varones mayores de 60 años, y están, en general, relacionados con el hábito tabáquico ${ }^{6,24-27}$. En nuestros pacientes, la edad media fue de 66 años, hubo también predominio de varones y relación con el tabaquismo (100\% fumadores).

No está claro el valor pronóstico de la DN. Se señala frecuentemente que la presencia de DN en los tumores de pulmón ensombrece el pronóstico de los pacientes $^{4,6,10-12}$. Aunque no todos los autores afirman que ésta por si misma sea un factor adverso ${ }^{28,}$ ${ }^{29}$, para algunos sería un factor pronóstico independiente junto con el performance status ${ }^{30}$. Otros autores opinan que la expresión de más de 2 marcadores neuroendocrinos sería más importante en términos de influencia sobre la supervivencia global, que la propia subclasificación histológica de los $\mathrm{TNP}^{31}$. Por último, es posible que niveles de algunos marcadores como la Enolasa Neuronal Específica, tengan valor pronóstico adverso ${ }^{32}$. Un trabajo reciente publicado por Takei ${ }^{11}$ correlaciona la supervivencia con el estadio de los TNP. Este estudio muestra una supervivencia a 5 años del 67\%, 75\%, 45\% y $0 \%$, para estadios I, II, III y IV respectivamente. Pero lo cierto es que debido a la rareza de estos tumores, poco se puede concluir sobre su pronóstico y su tratamiento óptimo ${ }^{33}$. Los datos de nuestra corta serie tampoco permiten sacar conclusiones sobre estos aspectos.

El tratamiento de los CNCG debe hacerse de forma similar al resto de $\mathrm{CPNCP}^{32}$, incluyendo, según los casos, la recomendación de no administrar radioterapia complementaria en los pacientes resecados de forma radical ${ }^{34,35}$, el uso de quimioterapia neoadyuvante ${ }^{36}$ o adyuvante ${ }^{37-41}$, o lo combinación de quimioterapia y radioterapia ${ }^{42}$. En general, debe indicarse cirugía para los CNCG en estadios localizados, pues aunque son tumores agresivos, su pronóstico mejora cuando se consigue una resección completa junto con una disección sistemática ganglionar mediastínica radical ${ }^{43,44}$. También hay experiencias con el uso de quimioterapia neoadyuvante, adyuvante y radioterapia ${ }^{43,45,46}$. En la serie de Iyoda, la quimioterapia adyuvante basada en cisplatino, prolongó la supervivencia de los CNCG en estadios iniciales ${ }^{47}$. En nuestra serie (Tabla IV), los dos pacientes tratados con quimioterapia adyuvante tuvieron supervivencias prolongadas. En tumores avanzados, se recomienda la utilización de quimioterapia e incluso octreotide $28-30,46,48,49$.

De acuerdo con algunas series, la DN podría asociarse con mayor riesgo de diseminación tumoral ${ }^{19}$, 48. Una cuestión diferente es si la presencia de DN confiere una capacidad diferente de respuesta a la quimioterapia ${ }^{9}$. De hecho otros autores señalan que los pacientes con CNCG tienen mayor supervivencia cuando reciben quimioterapia ${ }^{29,30,50}$. En nuestra serie, se observó respuesta objetiva a la quimioterapia tanto en esquemas de inducción como en enfermedad avanzada.

En conclusión, los CNCG representan menos del $5 \%$ de los CPNCP, y son de diagnóstico complejo sobre todo cuando no se dispone de una muestra suficiente de tejido tumoral. Los datos de nuestra serie corroboran las recomendaciones de otros autores en cuanto a que el manejo de los CNCG debe hacerse de forma similar al del resto de los CPNCP, con especial énfasis en la cirugía radical como tratamiento fundamental en los tumores localizados. No hay datos que indiquen una peor respuesta a los tratamientos de quimioterapia o radioterapia en este tipo de tumores.

Correspondencia:

Dr. R. García Gómez

Servicio de Oncología Médica

Hospital General Universitario Gregorio Marañón

C/ Doctor Esquerdo, 46

E-28007 Madrid 


\section{Bibliografía}

1. Shepherd FA, Carney DN. Treatment of NSCLC. Chemotherapy. En Textbook of Lung Cancer. International Association for the study of Lung Cancer. 213-242. Edited: Heine H Hansen. Martin Dunitz Ltd. 2000. The Livery House 7-9 Pratt Street. London. NW1. OAE .

2. Carney DN Shepherd FA. Treatment of SCLC: Chemotherapy. En Textbook of Lung Cancer. International Association for the study of Lung Cancer. 261-272. Edited: Heine H Hansen. Martin Dunitz Ltd. 2000. The Livery House 7-9 Pratt Street. London. NW1. OAE.

3. Arrigoni MG, Woolner LB, Bernatz PE. Atipical Carcinoid tumors of the lung. J Thorac Cardiovasc Surg. 1972; 64:413-421.

4. Travis W, Rush W, Flieder D, et al. Survival analysis of 200 Pulmonary Neuroendocrine Tumors with Clarification of Criteria for Atypical Carcinoid and its separation from Typical Carcinoid. Am J Surg Pathol. 1998; 22(8): 934944.

5. Travis W, Crrin B, Shimosato Y, Brambilla E. The Histological typing of lung and pleural tumours. WHO/ IASLC classification of lung and pleural tumours. 3rd ed. Berlin: Springer-Verlag; 1999.

6. Iyoda A, Hirosima K, Baba M, Saitoh Y, Ohwada H, Fujisawa T. Pulmonary Large Cell with Neuroendocrine Features are High-Grade Neuroendocrine Tumors. Ann Thorac Surg 2002; 73: 1049-1054.

7. Baylin SB. Neuroendocrine differenciación: A Pronostic Feature of Non-Small Cell Lung Cancer? Jour. Clin. Oncol. 1989; 7(10): 1375-1376.

8. Gazdar AF. Advances in the biology of lung Cancer. Clinical significance of Differentiation Neuroendocrine. Chest. 1989; 96(1) Supplement: 39S-41S.

9. Berendsen HH, De Leij L, Poppema S, et al. Clinical Characterization of Non-Small Cell Lung Cancer Tumours Showing Neuroendocrine Differentiation Features. Jour. Clin. Oncol. 1989; 7:1614-1620.

10. Dresler C, Ritter J, Patterson GA, Ross E, Bailey MS, Wick M. Clinical pathologic analysis of 40 patients with large cell neuroendocrine carcinoma of the lung. Ann Thorac Surg. 1997; 63:180-185.

11. Takei H, Asamura H, Maeshima A, et al. Large cell neuroendocrine carcinoma of the lung: A Clinicopathology study of eighty-seven cases. Jour. Thorac. And Cardi. Sur. 2002; 124(2):285-292.

12. Mountain CF. Revisions in the international system for staging lung cancer. Chest.1997; 111:1710-1717.

13. Therasse P, ArbacK SG, Einsenhauer EA, et al. New Guidlines to Evaluate the Response to Treatment in solid tumors. J Natl Cancer Inst. 2000; 92:205-16.

14. Brown WH. A case of a pluriglandular syndrome: Diabetes of bearded women. Lancet. 1928; II:1002-1003.

15. Younossian AB, Bründler MA, Tötsch M. Feasibility of the new WHO classification of pulmonary neuroendocrine tumours. Swiss. Med WKLY. 2002; 132:535-540.
16. Gugger M, Burckhardt E, Kappeler A, Hirsiger H, Laissue JA, Mazzuchelli L. Quantitative expansion of structural genomic alterations in the spectrum of neuroendocrine lung carcinomas. J Pathol. 2002; 196:408-415.

17. Addis BJ. Neuroendocrine differentiation in lung carcinoma. Thorax 1995; 50:113-115.

18. Carey FA, Save VE. Neuroendocrine differentiation lung cancer. J. Pathol. 1997; 182:9-10.

19. Sundaresan V, Reeve V, Stenning S, Stewart S, Bleehen NM. Neuroendocrine differentiation and clinical behaviour in non-small cell lung tumours. Br. J. Cancer. 1991; 64: 333-338.

20. Burnett HE, Spedding AV, Pendleton N, et al. Criteria for assessing the neuroendocrine phenotype and ist incidence in non-small cell lung cancer. Int J Oncol. 1993; 3:65-69.

21. Sturm N, Rossi G, Lanteujoul S, et al. Expression of Thyroid Transcription Factor-1 in the Spectrum of Neuroendocrine Cell Lung Proliferations wiht spectial interest in Carcinoids. Hum Pathol. 2002; 33:175-182.

22. Ullman R, Petzmann S, Klemen H, Fraire AE, Hasleton P, Popper HH. The position of Pulmonary Carcionoids within the spectrum of Neuroendocrine Tumors of the lung and other Tissues. Genes, Chrom \& CANCER 2002; 34:78-85.

23. Doddoli Ch, Berlasi F, Chetaille B, et al. Large Cell Neuroendocrine Carcinoma of the lung: An Agressive Disease Potentially Treatable with Surgery. Ann Thorac Surg. 2004; 77:1168-1172.

24. Flieder DB, Vazquez MF. Lung Tumors with Neuroendocrine Morphology. A perspective for the new millennium. Radio. Clin. Nort. Am. 2000; 38 (3):563- 577.

25. Huang Q, Muzitansky A, Mark E J. Pulmonary Neuroendocrine Carcinomas. A Review of 234 Cases and a Statistcal Analysis of 50 Cases Treated at One Institution Using a Simple Clinicopathologic Classification. Arch Pathol Lab Med 2002; 126:545-553.

26. García Gómez R, Texeira Fernández R, Jara Sánchez C, Gonzalez Beca R, Pérez Manga G. Carcinoide Bronquial. Tumor poco frecuente. Estudio Clínico de 12 casos. Rev Clin Esp. 1989; 185:9-13.

27. Schrevens L, Vansteenkiste J, Deneffe G, et al. Clinical-radiological presentation and outcome of surgically treated pulmonary carcinoid tumours: a long-term single institution experience. Lung Cancer 2004, 43:39-45.

28. Linnoila R I, Piantadosi S, Ruckdeschel J C. Impact of Neuroendocrine Differentiation in Non-small Cell Lung cancer. Chest 1994; 106(6):367S-371S.

29. Schleusener J T, Tazelaar H D, Jung S, et al. Neuroendocrine Differentiation is an Independent Prognostic Factor in Chemotherapy-Treated Nonsmall Cell Lung Carcinoma. Cancer 1996; 77:1284-1291.

30. Carles J, Rosell R, Ariza A, et al. Neuroendocrine differentiation as a prognostic factor in non-small cell lung cancer. Lung Cancer 1993; 10:209-219.

31. Harada M, Yokose T, Yoshida J, et al. Inmunohistochemical meuroendocrine differentiation is an independent prog- 
nostic factor in surgically resected large cell carcinoma of the lung. Lung Cancer 2002; 38:177-184.

32. Pujol JL, Boher JM, Grenier J, Quantin X. Cyfra-21, neuron specific enolase and prognostic of non-small cell lung cancer: Prospective study in 621 patients. Lung Cancer. 2001; 31:221-231.

33. Hage R, Seldenrijk K, de Bruin P, van Swieten H, van den Bosch J. Pulmonary large-cell neuroendocrine carcinoma (LCNEC). Eur J Cardiothorac Surg. 2003; 23:457-460.

34. PORT Meta-analysis Trialist Group (Stewart LA). Postoperative radiotherapy in non-small-cell lung cancer. Systematic review and meta-analysis of individual patient data from nine randomised controlled trials. Lancet 1998; 352: 257-263.

35. Dautzenberg B, Arriagada R, Chammard AB, et al. A controlled study of postoperative Radiotherapy for patients with completely resected Nonsmall Cell Lung Cancer. Cancer 1999; 86:265-273.

36. Lynch TJ. Treatment of Stage III Non-Small-Cell lung Cancer: 1998. Session Chair. A.S.C.O. Educational. 1998; 265-275.

37. Non-small Cell Lung Cancer Collaborative Group. Chemotherapy in non-small cell lung cancer: A meta-analisys using updated data on individual patients from 52 randomised clinical trials. Br Med J. 1995; 311:899-909.

38. Blum RH. Editorial. Adjuvant Chemotherapy for lung Cancer. A new standart of care. N Eng J Med 2004; 350: 404-405.

39. The International Adjuvant Lung Cancer Trial Collaborative Group: CisPlatin- Based Adjuvant Chemotherapy in Patients with Completely Resected. N Engl Med 2004; 350: 351-60.

40. Winton TL, Livingston R, Johnson D, et al. Vinorelbine plus cisplatin vs observation in resected non-samall cell lung cancer. N Eng J Med 2005; 352:2589-2597.

41. Douillard J, Rosell R, Delena M, Legroumellec A, Torres A, Carpagnano F. ANITA: Phase III adjuvant vinorelbine $(\mathrm{N})$ and cisplatin $(\mathrm{P})$ versus observation $(\mathrm{OBS})$ in comple- tely resected (stage I-III) non-small-cell lung cancer (NSCLC) patients (pts): Final results after 70-month median follow-up.On behalf of the Adjuvant Navelbine International Trialist Association. Proc Am Soc Clin Oncol 2005, Abstract 7013.

42. Choi NC. Management of Stage III Non-Small-Cell lung Cancer: Progress in Preoperative Chemoradiotherapy. Session Speaker. A.S.C.O. Educational. 1998; 276-289.

43. Zacharias J, Nicholson AG, Ladas GP, Goldstraw P. Large Cell Neuroendocrine Carcinoma with Neuroendocrine Morphology of the lung: Prognosis after Complete Resection and Systematic Nodal disection: Ann Thorac Surg. 2003; 75:348-352

44. García Yuste M, Matilla JM, Alvarez Gago T, et al. Pronostic factor in neuroendocrine lung tumors: A Spanish multicentre study. Ann Thorac Surg. 2000; 70:258-263.

45. Paci M, Cavazza A, Anessi V, et al. Large Cell Neuroendocrine Carcinoma of the Lung: A 10-Years Clinicopathologic Retrospective Study. Ann Thorac Surg. 2004; 77: 1163-1167.

46. Filosso PL. Large Cell Neuroendocrine Carcinoma (LCNC) of the lung: A dilema. Eur J Cardiothorac Surg. 2003; $24: 672$.

47. Iyoda A, Hirosima K, Toyozaki T, et al. Adyuvant Chemotherapy for Large Cell Carcinoma with Neuroendocrine Features. Cancer 2001; 92:1108-1112.

48. Mazières J, Daste G, Molinier L, et al. Large cell neuroendocrine carcinoma of the lung: Pathological study and clinical outcome of 18 resected cases. Lung Cancer 2002; 37: 287-292.

49. Gazdar AF, Kadoyama C, Venzon D, et al. Association between histological type and neuroendocrine differentiation on drug sensitivity of lung cancer cell lines. J Nat Cancer Institute. Monographs. 1992; 13:191-196.

50. Graciano SL, Mazid R, Newman N, et al. The use of neuroendocrine immunoperoxidase markers to predict Chemotherapy response in patiens with non-small cell lung cancer. J Cin Oncol. 1989; 7:1398-1406. 